


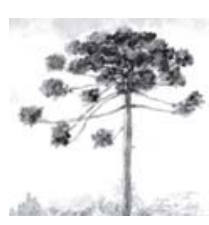

\title{
ASPECTOS BIOLÓGICOS DE DUAS ESPÉCIES DE Astyanax (TELEOSTEI, CHARACIDAE) EM LAGOAS MARGINAIS DO ALTO RIO IGUAÇU, PARANÁ, BRASIL
}

\author{
Biological aspects of two Astyanax species (TELEOSTEI, CHARACIDAE) \\ in marginal ponds of the upper Iguacu river, Parana, Brazil
}

\section{Vinícius Abilhoa ${ }^{1}$, Angelo Antonio Agostinho²}

\footnotetext{
${ }^{1}$ Pesquisador, GPIc - Grupo de Pesquisas em Ictiofauna. Museu de História Natural Capão da Imbuia, Prefeitura de Curitiba, Curitiba, PR - Brasil, e-mail: vabilhoa@uol.com.br

${ }^{2}$ Professor Doutor, Núcleo de Pesquisas em Limnologia, Ictiologia e Aqüicultura (Nupélia), Universidade Estadual de Maringá. Maringá, PR - Brasil, e-mail: agostinhoaa@nupelia.uem.br
}

\begin{abstract}
Resumo
Aspectos da biologia de duas espécies endêmicas de Astyanax da bacia do rio Iguaçu são descritos. Amostragens mensais em lagoas marginais do curso superior do rio Iguaçu foram realizadas entre julho de 1995 e junho de 1996, dentro do Parque Municipal do Iguaçu. Foram capturados 2.154 exemplares, sendo 46\% pertencentes à Astyanax sp. B e 54\% a Astyanax sp. C. Variações significativas nas médias de comprimento e peso totais foram observadas, com valores maiores durante verão/outono para Astyanax sp. B e inverno/primavera para Astyanax sp. C. O período reprodutivo das espécies foi longo, com maior intensidade na primavera e verão, quando a proporção sexual foi predominantemente 1:1. Variações dos aspectos biológicos estiveram relacionadas às alterações sazonais do ambiente.
\end{abstract}

Palavras-chave: Estrutura populacional; Reprodução; Fator de condição.

\begin{abstract}
Biological aspects of two endemic Astyanax species of the Iguaçu river are described. Monthly samples in marginal ponds of the upper Iguaçu river were taken from July 1995 to June 1996, into the Iguaçu Municipal Park. A total of 2,154 specimens were captured, $46 \%$ of Astyanax sp. B and $54 \%$ of Astyanax sp. C. Significant variations in the average values of length and weight were observed, with higher values in summer/autumn for Astyanax sp. B and winter/spring for Astyanax sp. C. The reproductive period was long, with higher intensity in spring and summer, when the sex ratio was mostly 1:1. Variations in the biological aspects were related to seasonal alterations in the environment.
\end{abstract}

Keywords: Population structure; Reproduction; Condition factor. 


\section{INTRODUÇÃO}

As cavas formadas pelas atividades de mineração de areia e argila para a construção civil, em conjunto com o leito meandrante dos rios e com as lagoas marginais naturais da planície aluvial do alto curso do rio Iguaçu, formam um sistema de canais anastomosados, que conferem um aspecto peculiar à região (1).

Esse complexo de unidades naturais e artificiais forma uma paisagem com características próprias e o comportamento das espécies aquáticas frente aos fatores físicos e químicos deste ambiente ainda não é conhecido. Como já descrito para áreas naturalmente alagáveis, este tipo de ecossistema apresenta elevada flutuação de fatores hidrológicos e limnológicos, os quais exercem grande influência sobre a disponibilidade de alimento e abrigo para a fauna aquática $(2,3,4,5)$. Na bacia do rio Iguaçu, estes ambientes são favoráveis à propagação de inúmeras espécies de peixes, como os lambaris do gênero Astyanax (Characidae).

Os primeiros levantamentos realizados nestes ambientes registraram duas espécies ainda não formalmente descritas na literatura: Astyanax sp. B e Astyanax sp. C. Esta nomenclatura foi proposta por Sampaio (6), e vem sendo utilizada por diversos autores $(7,8)$.

Este trabalho foi realizado com o objetivo de entender o comportamento destas espécies frente às características deste ambiente aquático, gerando informações que permitam a avaliação dos hábitats marginais e da influência de alguns fatores físicos e químicos sobre a ictiofauna regional.

\section{MATERIAIS E MÉTODOS}

Os exemplares de Astyanax foram obtidos por meio de amostragens mensais realizadas entre julho de 1995 e junho de 1996 no Parque Municipal do Iguaçu (Figura 1). Este Parque situa-se na região sudeste do Município de Curitiba e é composto por cavas desativadas, meandros abandonados do rio Iguaçu, várzeas alteradas e um grande canal artificial para controle das cheias. A fitofisionomia é formada pela Floresta Ombrófila Mista Aluvial, que corresponde às florestas de galeria ou ciliares ligadas aos rios, entremeadas por várzeas graminóides úmidas. Grandes bancos de macrófitas, principalmente de Eichhornia sp. e Pistia sp., são encontrados na região litorânea das lagoas.

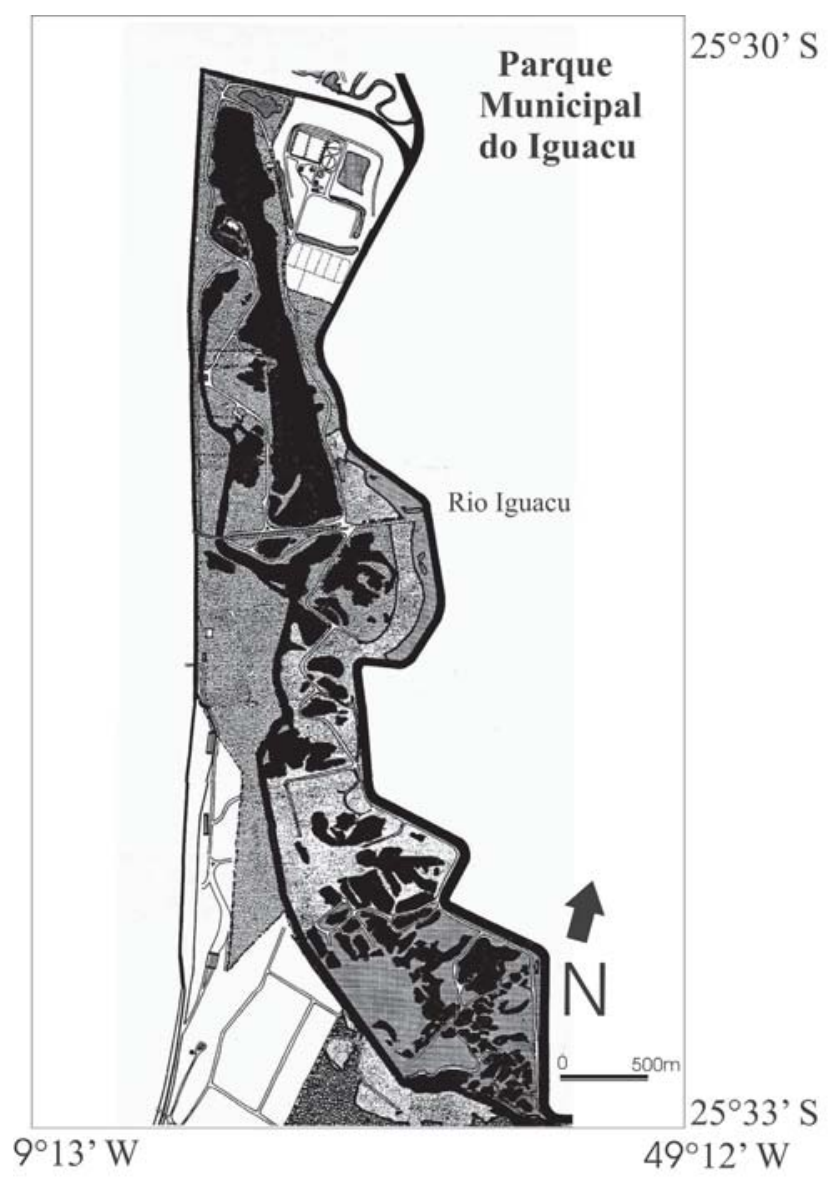

FIGURA 1 - Área de amostragem no Parque Municipal do Iguaçu, na região sudeste do Município de Curitiba, composta por cavas desativadas, meandros abandonados do rio Iguaçu, várzeas alteradas e um grande canal artificial para controle das cheias.

Esta unidade de conservação está incluída na Área de Proteção Ambiental do Iguaçu (9) e ocupa porções das regiões leste, sudoeste e sul do Município de Curitiba, estando situada entre as coordenadas $25^{\circ} 25^{\prime}$ e $25^{\circ} 38^{\prime}$ de latitude sul e $49^{\circ} 12^{\prime}$ e $49^{\circ} 21^{\prime}$ de longitude oeste.

Para as coletas, foram utilizados esforços constantes durante todo o período amostral, sendo que as redes de espera permaneceram 24 horas na água. Os equipamentos de captura empregados foram três redes de espera simples de 20 metros de comprimento, sendo duas com malhas de $1 \frac{1 / 2}{2}$ 
$\mathrm{cm}$ e uma com $2^{1 / 2} \mathrm{~cm}$ entre nós consecutivos, além de lances padronizados de redes de arremesso (tarrafa, malha de $1 \frac{1}{2} \mathrm{~cm}$ ). Os espécimes amostrados foram colocados em sacos plásticos etiquetados contendo solução de formol $4 \%$. Também foram obtidos dados de temperatura do ar e da água, $\mathrm{pH}$ (pHmetro digital) e oxigênio dissolvido (oxímetro digital) para cada estação do ano durante o período de estudo pelo Laboratório de Análise de Água do MHNCI Museu de História Natural Capão da Imbuia da Prefeitura de Curitiba.

Em laboratório, os exemplares foram identificados, contados e medidos (comprimento total; CT), pesados (peso total) e dissecados para a observação macroscópica do sexo e estádio de maturação gonadal, esta última com base em escala proposta por Vazzoler (10). A determinação dos estádios de maturação foi baseada no tamanho dos exemplares e em características como coloração das gônadas, transparência, irrigação sanguínea e presença, no caso dos ovários, de ovócitos visíveis a olho nu.

Para a análise dos dados de estrutura populacional em tamanho, a distribuição de freqüência das classes de comprimento total para ambos os sexos das espécies capturadas foi obtida pela regra de Sturge (11).

Para a avaliação da influência da estação do ano (fator) sobre os valores médios das capturas em tamanho e peso foram realizados testes de análise de variância unifatorial, tendo em vista que os pressupostos de homogeneidade das variâncias (teste de Bartlett) e normalidade (teste de Shapiro-Wilk) foram atendidos pelos dados. Os valores de comprimento total (CT) e peso foram então expressos por médias e desvio-padrão das estimativas $( \pm s)$.

A relação entre o peso e o comprimento total foi obtida para machos e fêmeas das espécies analisadas, sendo expressa pela equação: Peso $=a$ $x$ Comprimento total , onde os parâmetros $a$ e $b$ foram obtidos ajustando-se o logaritmo das variáveis dependente e independente pelo método dos mínimos quadrados $(12,13)$. O valor $b$ (inclinação) é a constante de crescimento relativo e tende a assumir valores próximos a 3 , expressando um crescimento isométrico, ou seja, com taxas iguais de incremento em diferentes partes do corpo (14). Entretanto, esta relação cúbica nem sempre é obedecida, podendo, segundo Pauly (15), ser abaixo de 3 (alométrico negativo) ou acima de 3 (alométrico positivo). Os valores do fator de condição foram calculados para cada indivíduo amostrado por meio da equação: $\mathrm{K}=\mathrm{Peso} /$ Comprimento total ${ }^{\mathrm{b}}(12,13)$, sendo que as diferenças entre os valores médios para cada parâmetro considerado foram avaliadas pelo teste de Kruskall-Wallis (16), tendo em vista que os valores não apresentaram distribuição normal segundo o teste de Shapiro-Wilk.

A proporção sexual foi determinada mediante distribuições mensais das freqüências de machos e fêmeas ao longo do período de estudo. Utilizou-se o teste de qui-quadrado $\left(X^{2}\right.$; $a=0,05)$ para a verificação de eventuais diferenças significativas nestas proporções. A distribuição de freqüência dos estádios de maturação gonadal das fêmeas foi utilizada para determinar o período reprodutivo (13).

Os testes estatísticos (Anova, quiquadrado, Shapiro-Wilk e Kruskall Wallis) foram realizados com emprego do programa Statistica, 5.1 (17), sendo que as diferenças implicaram em nível de significância de 5\%.

\section{RESULTADOS}

\section{Caracterização da área estudada}

Segundo os dados da rede hidrometeorológica do Instituto Tecnológico SIMEPAR, as maiores precipitações ocorreram entre os meses de novembro/1995 e março/1996, e as maiores temperaturas foram observadas entre os meses de janeiro e março de 1996. O clima da região é considerado temperado-úmido, sem uma estação seca definida, com temperaturas médias inferiores a $22^{\circ} \mathrm{C}$ para o mês mais quente e temperaturas médias superiores a $10^{\circ} \mathrm{C}$ na maior parte do ano (18).

A temperatura do ambiente e as concentrações de oxigênio dissolvido variaram sazonalmente ao longo do período amostral, sendo os maiores valores observados no verão, período de maior precipitação. Os valores de $\mathrm{pH}$ não apresentaram grandes variações entre as quatro estações analisadas, situando-se próximos à neutralidade, com oscilações entre 6,8 e 7,8 (Tabela 1). 
TABELA 1 - Temperatura do ambiente $\left({ }^{\circ} \mathrm{C}\right)$, temperatura da água $\left({ }^{\circ} \mathrm{C}\right)$, potencial hidrogeniônico $(\mathrm{pH})$ e oxigênio dissolvido $(\mathrm{OD} ; \mathrm{mg} / \mathrm{L})$ registrados uma única vez em cada estação do ano durante as amostragens no Parque Municipal do Iguaçu, no Paraná, entre julho 1995/junho 1996

\begin{tabular}{ccccc}
\hline ESTAÇÃO & TEMP. AMBIENTE & TEMP. ÁGUA & pH & OD \\
\hline Inverno & $12^{\circ} \mathrm{C}$ & $14,5{ }^{\circ} \mathrm{C}$ & 6,89 & 5,81 \\
Primavera & $22^{\circ} \mathrm{C}$ & $21,5{ }^{\circ} \mathrm{C}$ & 7,17 & 4,35 \\
Verão & $24^{\circ} \mathrm{C}$ & $22^{\circ} \mathrm{C}$ & 7,19 & 6,73 \\
Outono & $16^{\circ} \mathrm{C}$ & $16^{\circ} \mathrm{C}$ & 7,82 & 6,39 \\
\hline
\end{tabular}

\section{Abundância e estrutura em comprimento e peso}

Foram capturados 2.154 indivíduos, sendo $985(45,7 \%)$ pertencentes à espécie Astyanax sp. B e 1.169 (54,3\%) à espécie Astyanax sp. C. As freqüências de Astyanax sp. C e Astyanax sp. B nos diferentes meses de captura na área de estudo são mostradas na Figura 2. As espécies apresentaram maiores freqüências de captura entre janeiro e abril de 1996 (verão-outono).

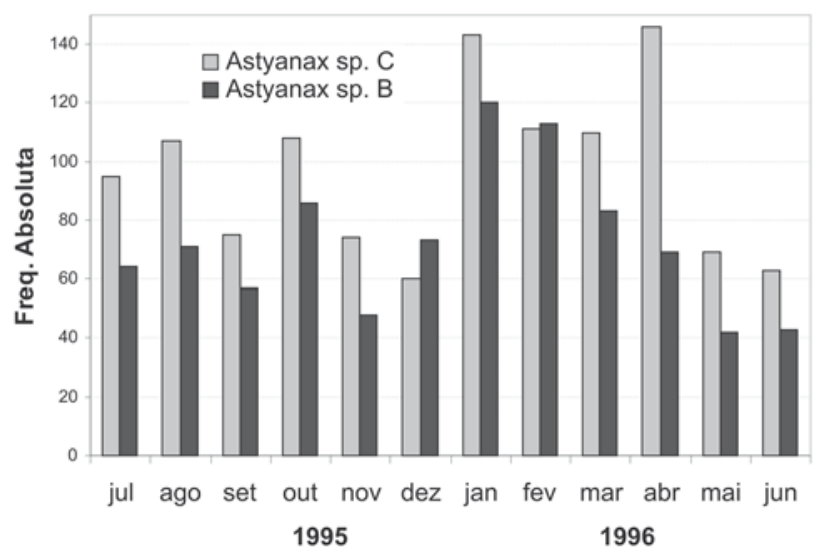

FIGURA 2 - Freqüência absoluta de exemplares de Astyamax sp. B e Astyamax sp. C capturados mensalmente na área estudada no Parque Municipal do Iguaçu, Região Metropolitana de Curitiba, Paraná, entre os anos de 1995 e 1996

A distribuição de freqüência por classe de comprimento total para ambos os sexos revelou que os valores modais foram semelhantes para ambas as espécies, sendo mais abundantes entre os comprimentos de 9,0 e $10,3 \mathrm{~cm}$. No entanto, os exemplares de Astyanax sp. B alcançaram maior comprimento total. A distribuição de comprimento total por classes revelou também que as capturas de Astyanax sp. B e Astyanax sp. C foram mais freqüentes na classe de 9,0 - 9,6 cm (Figura 3).

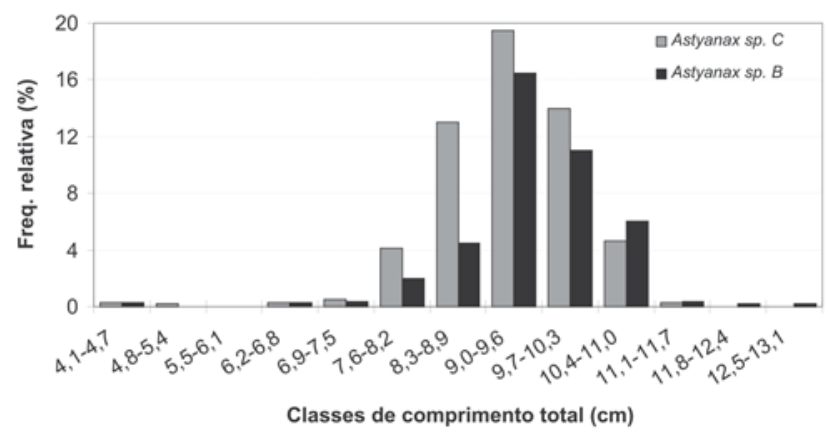

FIGURA 3 - Distribuição de freqüência de exemplares de Astyamax sp. B e Astyamax sp. C por classe de comprimento, nas capturas mensais realizadas na área do Parque Municipal do Iguaçu, Região Metropolitana de Curitiba, Paraná, entre os anos de 1995 e 1996

A amplitude do comprimento total variou de 4,6 até $12,9 \mathrm{~cm}$, sendo que a média de comprimento total de Astyanax sp. C $(9,26 \pm 0,82$ $\mathrm{cm})$ foi menor que a de Astyanax sp. B $(9,51 \pm 0,95$ $\mathrm{cm}) \quad(\mathrm{F}=35,99 ; \quad \mathrm{p}<0,05), \quad$ com valores significativamente maiores durante verão/outono para Astyanax sp. B e inverno/primavera para Astyanax sp. C (Tabela 2).

Variações significativas nas médias de comprimento total foram observadas para Astyanax sp. B $(\mathrm{F}=3,521 ; \mathrm{p}<0,05)$ e Astyanax sp. C $(\mathrm{F}=19,65 ; \mathrm{p}<0,05)$. Somente Astyanax sp. C apresentou diferenças significativas nas médias sazonais de peso $(\mathrm{F}=16,39 ; \mathrm{p}<0,05)$ durante as amostragens realizadas entre os meses de julho de 1995 e junho de 1996 (Tabela 2). 
Aspectos biológicos de duas espécies de Astyanax (TELEOSTEI, CHARACIDAE) em lagoas marginais de alto Rio Iguaçu, Paraná, Brasil

TABELA 2 - Variações sazonais nos comprimentos e pesos totais médios de Astyanax sp. B e Astyanax sp. C de exemplares capturados no Parque Municipal do Iguaçu, Paraná - julho 1995/junho 1996. $(*=\mathrm{p}<0,05)$

\begin{tabular}{lcccc}
\hline & \multicolumn{2}{c}{ COMPRIMENTO TOTAL (cm) } & \multicolumn{2}{c}{ PESO (g) } \\
& Astyanax sp. B & Astyanax sp. C & Astyanax sp. B & Astyanax sp. C \\
\hline Inverno & $9,36 \pm 1,49$ & $9,28 \pm 0,88^{*}$ & $10,35 \pm 4,21$ & $10,38 \pm 3,46$ \\
Primavera & $9,33 \pm 0,89$ & $9,53 \pm 0,69^{*}$ & $9,54 \pm 2,69$ & $10,79 \pm 2,66^{*}$ \\
Verão & $9,62 \pm 0,69^{*}$ & $9,24 \pm 0,87$ & $9,86 \pm 2,36$ & $9,69 \pm 2,79$ \\
Outono & $9,67 \pm 0,65^{*}$ & $9,08 \pm 0,74$ & $10,24 \pm 2,16$ & $9,66 \pm 2,65$ \\
\hline
\end{tabular}

\section{Relação entre peso e comprimento total}

As relações entre o peso e o comprimento total para machos e fêmeas das espécies Astyanax sp. $\mathrm{B}$ e Astyanax sp. C, assim como as equações que as descrevem, estão representados na Figura 4. Os valores do parâmetro b variaram entre 2,6 (machos de Astyanax sp. C) e 3,0 (fêmeas de Astyanax sp.C). Os menores valores foram encontrados para os machos de ambas as espécies.

\section{Astyanax sp. B}
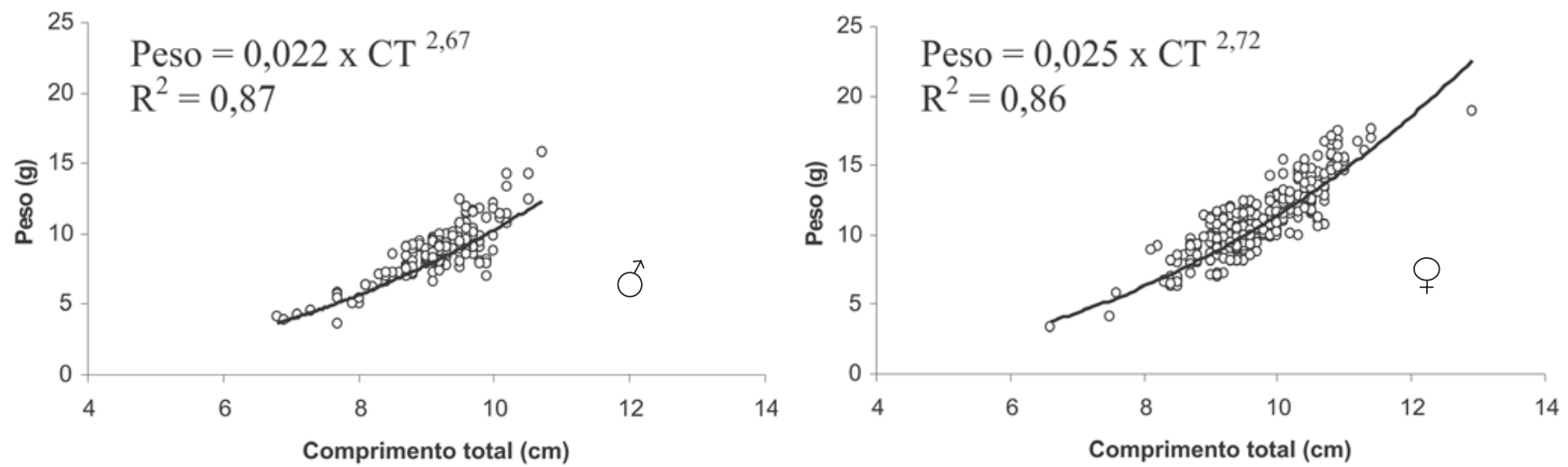

\section{Astyanax sp. C}
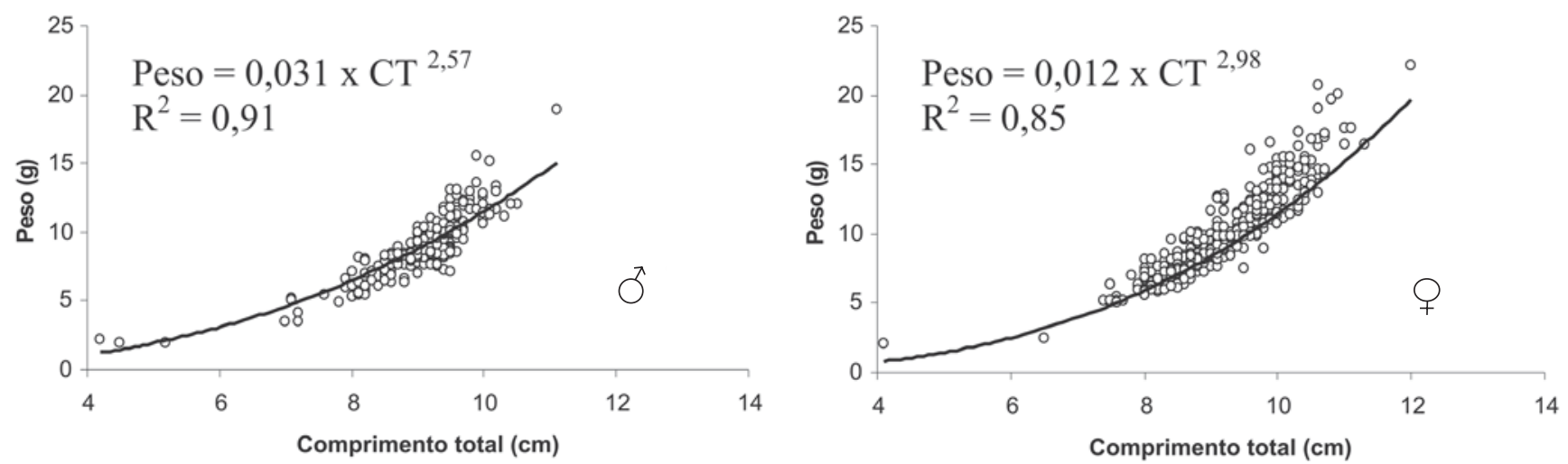

FIGURA 4 - Representação dos ajustes da relação peso-comprimento total para machos e fêmeas de Astyanax sp. B e Astyanax sp. C capturados mensalmente na área estudada no Parque Municipal do Iguaçu, Região Metropolitana de Curitiba, Paraná, entre os anos de 1995 e 1996. São também representadas as equações da relação e os coeficientes de determinação $\left(R^{2}\right)$ 


\section{Fator de condição}

O fator de condição foi obtido para cada indivíduo por meio das relações peso/ comprimento obtidas para machos e fêmeas. Como os valores não apresentaram distribuição normal para Astyanax sp. B (Shapiro-Wilk W test $=$ 0,991; $\mathrm{p}<0,05)$ e Astyanax sp. C (Shapiro-Wilk $W$ test $=0,806 ; \mathrm{p}<0,05)$, as médias foram avaliadas pelo teste não-paramétrico de Kruskall-Wallis, sendo que os limites de confiança foram utilizados para a identificação de diferenças significativas.

Foram encontradas diferenças significativas para os valores do fator de condição entre machos e fêmeas de Astyanax sp. B $(\mathrm{H}=68,89 ; \mathrm{p}<0,05)$ e Astyanax sp. $\mathrm{C}(\mathrm{H}=438,43$; $\mathrm{p}<0,05)$, sendo que as fêmeas de Astyanax sp. B apresentaram maiores médias. Com relação às variações sazonais, somente Astyanax sp. C apresentou diferenças $(\mathrm{H}=18,69 ; \mathrm{p}<0,05)$, com valores médios menores no inverno e maiores no período primavera/verão.

\section{Proporção sexual}

A proporção sexual durante o período de estudo está representada na Figura 5. Para Astyanax sp. C observou-se um predomínio significativo de fêmeas durante os meses de julho $\left(X^{2}=11,6\right.$; $\mathrm{p}<0,05)$, agosto $\left(X^{2}=23,5 ; \mathrm{p}<0,05\right)$ e setembro $\left(X^{2}=12,5 ; \mathrm{p}<0,05\right)$ de 1995 , e abril $\left(X^{2}=20,3\right.$; $\mathrm{p}<0,05)$, maio $\left(X^{2}=25,1 ; \mathrm{p}<0,05\right)$ e junho $\left(X^{2}=\right.$ $82,6 ; \mathrm{p}<0,05)$ de 1996. Além desses períodos, freqüências maiores de fêmeas foram registradas durante o verão no mês de fevereiro de $1996\left(X^{2}=\right.$ 9,8; $\mathrm{p}<0,05)$, e somente em novembro de 1995 a freqüência de machos foi maior $\left(X^{2}=7,5 ; \mathrm{p}<0,05\right)$.

A

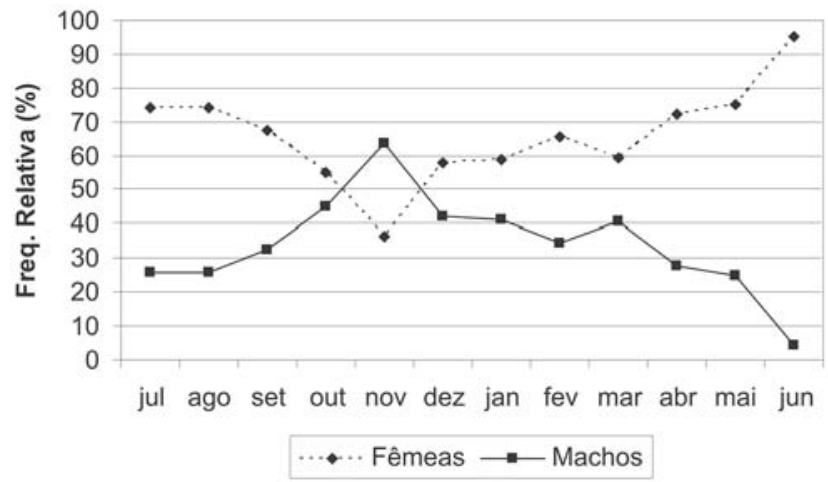

B

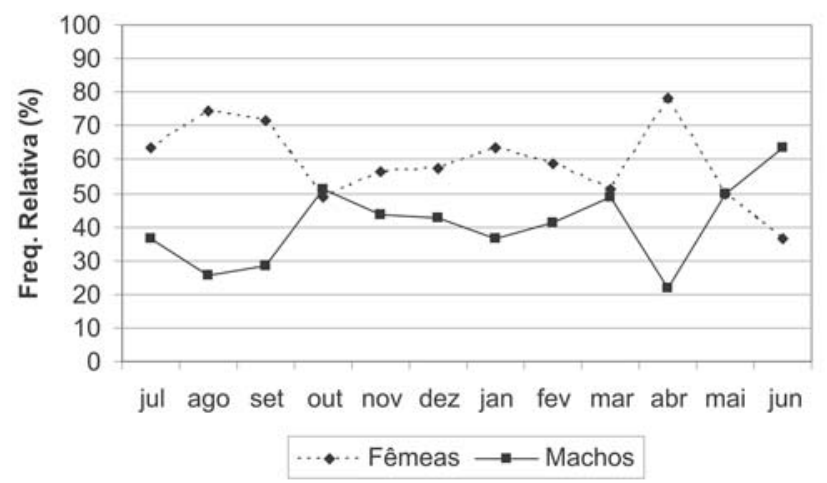

FIGURA 5 - A e B - Proporção entre machos e fêmeas de Astyanax sp. C (A) e Astyanax sp. B (B) capturados mensalmente na área estudada no Parque Municipal do Iguaçu, Região Metropolitana de Curitiba, Paraná, entre os anos de 1995 e 1996

Resultados semelhantes foram observados para a espécie Astyanax sp. B, que também apresentou um predomínio significativo de fêmeas durante os meses de julho $\left(X^{2}=7,1 ; \mathrm{p}<0,05\right)$, agosto $\left(X^{2}=23,7\right.$; $\mathrm{p}<0,05)$ e setembro $\left(X^{2}=18,4 ; \mathrm{p}<0,05\right)$ de 1995 , e abril $\left(X^{2}=32,2 ; \mathrm{p}<0,05\right)$ de 1996 , sendo que somente em junho $\left(X^{2}=7,4 ; \mathrm{p}<0,05\right)$ de 1996 a captura de machos foi maior. Entre outubro de 1995 e março de 1996, incluindo também o mês de maio deste mesmo ano, não foram observadas diferenças significativas nas freqüências de fêmeas e machos.

\section{Período reprodutivo}

As distribuições de freqüência dos estádios de desenvolvimento gonadal das fêmeas de Astyanax sp. B e Astyanax sp. C estão mostradas na Figura 6. Fêmeas maduras ocorreram em todo período amostral, enquanto que gônadas imaturas e em descanso foram pouco representativas.

O período reprodutivo das espécies parece ser longo, mas a atividade foi maior na primavera e no verão, onde um maior número de indivíduos com ovários maduros foi capturado. 

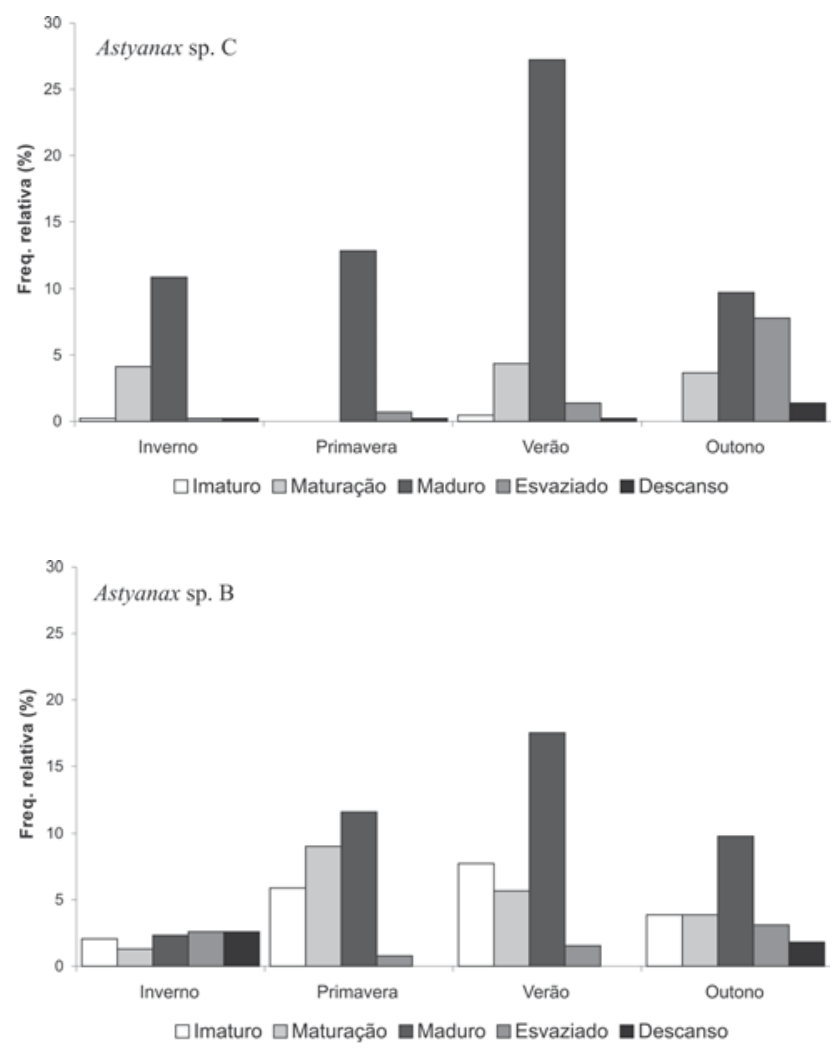

FIGURA 6 - Freqüência relativa sazonal dos estádios de maturação dos ovários de Astyanax sp. C e Astyanax sp. B capturados mensalmente na área estudada no Parque Municipal do Iguaçu, Região Metropolitana de Curitiba, Paraná, entre os anos de 1995 e 1996

\section{DISCUSSÃO}

Os lambaris estudados no Parque Municipal do Iguaçu, em um ambiente aquático composto por cavas desativadas, lagoas marginais, meandros abandonados do rio Iguaçu e várzeas alteradas, mostraram que os valores médios de peso, comprimento, dos parâmetros da relação pesocomprimento e do período reprodutivo apresentaram variações ao longo do período amostral.

Para os exemplares analisados, desconsiderando-se a seletividade dos aparelhos de pesca utilizados, foi verificado que as espécies apresentaram semelhanças nas alterações da abundância e na estrutura em tamanho, muito embora Astyanax sp. B tenha atingido comprimentos maiores.

Ambas as espécies apresentaram maior freqüência numérica durante a primavera e verão, período de maior precipitação, o que pode ter reflexo (i) no crescimento, (ii) no recrutamento, ou (iii) na maior disponibilidade de fontes alimentares.

Diferenças nos valores dos parâmetros $\mathbf{b}$ e $\mathbf{k}$ obtidos por meio das relações peso/comprimento foram observadas entre os sexos para ambas as espécies, sugerindo um desenvolvimento diferenciado. O fator de condição apresentou diferenças significativas entre os sexos e a espécie Astyanax sp. C também apresentou diferenças entre as estações do ano para este parâmetro. De acordo com diversos autores, essas diferenças são interpretadas como medidas de várias características biológicas, podendo estar relacionadas ao tamanho das gônadas (19), visto que o fator de condição pode ser considerado como um indicador de desova $(20,21)$.

A atividade reprodutiva das espécies analisadas foi maior na primavera e no verão, coincidindo com o observado para outras espécies na bacia do Paraná $(22,23)$ e para as mesmas espécies no reservatório de Segredo no médio rio Iguaçu (24), com maior freqüência de fêmeas em atividade reprodutiva entre outubro e março.

Muito embora a análise macroscópica permita falhas na eventual diferenciação de indivíduos em maturação e maduros, esse longo período reprodutivo pode indicar um mecanismo de desova parcelada (10), em acordo com o verificado para outras espécies de Astyanax (25, 26, 27, 28).

Essa tática reprodutiva é importante para o sucesso das espécies nesse tipo de ambiente, onde a manutenção de populações viáveis é garantida por um processo reprodutivo longo, em função de um ambiente com severas alterações nos níveis fluviométricos e nas condições físicas e químicas, como nas planícies alagáveis (23).

A proporção sexual foi de 1:1 praticamente durante todo período reprodutivo (outubro-março), porém com um leve predomínio das fêmeas, as quais durante o inverno e o outono foram significativamente mais freqüentes nas capturas de ambas as espécies. Variações na proporção entre machos e fêmeas são comuns na bibliografia (29, 30, 31, 32), inclusive para Astyanax (25), o que pode estar relacionado a diversos fatores, como taxas de crescimento diferenciado, maior mortalidade dos machos, maior longevidade das fêmeas ou até mesmo à suscetibilidade aos aparelhos de pesca utilizados.

As espécies de Astyanax têm uma ampla distribuição geográfica na bacia do rio Iguaçu, o que leva a crer que sua abundância registrada em 
reservatórios $(33,34,35)$ e na área de estudo é decorrente da tolerância que estes organismos apresentam às variações ambientais.

Em uma análise geral, se considerarmos o pequeno porte, o longo período reprodutivo, o amplo espectro alimentar e a tolerância ambiental, é possível caracterizarmos as espécies estudadas de Astyanax como oportunistas, as quais, segundo Agostinho e Gomes (8), exibem maior facilidade para a invasão de novos ambientes. Essas características adaptativas permitem que essas espécies sobrevivam em condições estressantes, como as que ocorrem nas lagoas marginais distribuídas ao longo das várzeas do alto curso do rio Iguaçu.

\section{REFERÊNCIAS}

1. Pellenz E, Loyola LC. A extração mineral como objetivo de análise do uso do solo na área dos mananciais do Alto Iguaçu / Região Metropolitana de Curitiba / Paraná. In: $1^{\circ}$ Simpósio Sul Americano, 1 e Anais do 2 Simpósio Nacional de Recuperação de Áreas Degradadas. Foz do Iguaçu, Brasil. Curitiba; 1994. p. 211-224.

2. Winemiller KO. Ontogenetic diet shifts and resource partioning among piscivorous fishes in the Venezuelan llanos. Environmental Biology of Fishes. 1990; 26:177-199.

3. Esteves KE. Feeding ecology of three Astyanax species (Characidae, Tetragonopterinae) from a floodplain lake of MogiGuaçu River, Paraná River Basin, Brazil. Environmental Biology of Fishes. 1996; 46:83-101.

4. Kattelmann R, Embury M. Riparian areas and wetlands. Davis: Centers for Water and Wildland Resources; 1996.

5. Harper D, Mekotova J, Hulme S, White J, Hall J. Habitat heterogeneity and aquatic invertebrate diversity in floddplain forests. Global Ecology and Biogeography Letters. 1997;6:275-285.
6. Sampaio FAA. Estudos taxonômicos preliminares dos Characiformes (Teleostei, Ostariophysi) da bacia do rio Iguaçu, com comentários sobre o endemismo dessa fauna [dissertação]. São Carlos: Universidade Federal de São Carlos; 1988.

7. Severi W, Cordeiro AAM. Catálogo de peixes na Bacia do Rio Iguaçu. Curitiba: Instituto Ambiental do Paraná/GTZ; 1994.

8. Agostinho AA, Gomes LC. Reservatório de Segredo: bases ecológicas para o manejo. Maringá: EDUEM; 1997.

9. Curitiba. Decreto Municipal n 410 de 15/ 07/1991. Institui a Área de Proteção Ambiental do Iguaçu. Diário Oficial do Município.

10. Vazzoler AEAM. Biologia da reprodução de peixes telósteos: teoria e prática. Maringá: EDUEM; 1996.

11. Vieira S. Introdução à bioestatística. Rio de Janeiro: Campus; 1980.

12. Santos EP. Dinâmica de populações aplicada a pesca e piscicultura. São Paulo: EDUSP; 1978.

13. Vazzoler AEAM. Manual de métodos para estudos biológicos de populações de peixes; reprodução e crescimento. Brasília: CNPq; 1981.

14. Weatherley AH, Gill HS. The biology of fish growth. London: Academic Press; 1987.

15. Pauly D. Editorial: fishbyte section. The ICLARMQ. 1993;16(2/3):1-26.

16. Vieira S. Bioestatística: tópicos avançados. Rio de Janeiro: Campus; 2003.

17. Statsoft. Electronic statistics textbook. Tulsa: Statsoft; 1999.

18. Iapar - Instituto Agronômico do Paraná. Cartas climáticas básicas do Estado do Paraná. Londrina: Iapar; 1978. 
19. Benedito-Cecílio E, Agostinho AA. Estrutura das populações de peixes do reservatório de Segredo. In: Agostinho AA, Gomes LC. Reservatório de Segredo: bases ecológicas para o manejo. Maringá: EDUEM; 1997. p. 113-139.

20. Barbieri G, Verani JR. O fator de condição como indicar do período de desova em Hypostomus aff. plecostomus (Linnaeus, 1758) (Osteichthyes, Loricariidae) na represa do Monjolinho (São Carlos, SP). Ciência e Cultura. 1987;39:655-658.

21. Goulart E, Verani JR. Proporção sexual, relação peso/comprimento e fator de condição em Hypostomus commersonii Valenciennes, 1840 (Osteichthyes, Loricariidae) da represa de Capivari Cachoeira, Paraná, Brasil. Unimar. 1992;14(supl):19-33.

22. Vazzoler AEAM, Menezes NA. Síntese dos conhecimentos sobre o comportamento reprodutivo dos Characiformes $\mathrm{da}$ América do Sul (Teleostei, Ostariophysi) Revista Brasileira de Biologia. 1992; 52(4):626-640.

23. Vazzoler AEAM, Suzuki HI, Marques EE, Lizama MAP. Primeira maturação gonadal, períodos e áreas de reprodução. In: Vazzoler AEAM, Agostinho AA, Hahn NS. A planície de inundação do alto rio Paraná: aspectos físicos, biológicos e socioeconômicos. Maringá: EDUEM; 1997. p. 249-280.

24. Suzuki HI, Agostinho AA. Reprodução de peixes do reservatório de Segredo. In: Agostinho AA, Gomes LC. Reservatório de segredo: bases ecológicas para o manejo. Maringá: EDUEM; 1997. p. 163-182.

25. Nomura H. Fecundidade, maturação sexual e índice gonadossomático de lambaris do gênero Astyanax Baird \& Girard, 1854 (Osteichthyes, Characidae) relacionados com fatores ambientais. Revista Brasileira de Biologia. 1975;34(4):775-798.
26. Barbieri G, Barbieri MC. Curva de maturação, tamanho de primeira maturação gonadal e fecundidade de Astyanax bimaculatus e Astyanax fasciatus da represa do Lobo, Estado de São Paulo (Osteichthyes, Characidae). Ceres. 1988;35(197):64-77.

27. Garutti V. Contribuição ao conhecimento reprodutivo de Astyanax bimaculatus (Ostariophysi, Characidae) em cursos de água da bacia do rio Paraná. Revista Brasileira de Biologia. 1989;49(2):489-495.

28. Barbieri G. Biologia de Astyanax scaripinnis paranae (Characiformes, Characidae) do Ribeirão do Fazzari. São Carlos. Estado de São Paulo. II. Aspectos quantitativos da reprodução. Revista Brasileira de Biologia. 1992;52(4):589-596.

29. Gomes AL, Monteiro FP. Estudo da população total de peixes da represa da estação experimental de biologia e piscicultura, em Pirassununga, São Paulo. Revista de Biologia Marinha. 1955; 6(1/3):82-133.

30. Gurgel HCB. Estrutura da população de Metynnis cf. roosevelti Eigenmann, 1915 (Characidae, Myleinae) da Lagoa Redonda, Nízia Floresta, Rio Grande do Norte Brasil. Boletim do Instituto de Pesca. 1995; 22(1):61-71.

31. Schifino LC, Fialho CB, Verani JR. Reproductive aspects of Cyphocharax voga (Hensel) from Custódias Lagoon, Rio Grande do Sul, Brazil (Characiformes, Curimatidae). Revista Brasileira de Zoologia. 1998;15(3):767-773.

32. Takeuti DF, Verani JR, Aranha JMR, Menezes MS. Population structure and condition factor of Pseudotothyris obtusa (Hypoptopomatinae) from three coastal streams in southern Brazil. Brazilian Archives of Biology an Technology. 1999; 42(4):397-403. 
33. Agostinho AA, Ferretti CML, Gomes LC, Hahn, NS, Suzuki HI, Fugi R, Abujanra F. Ictiofauna de dois reservatórios do rio Iguaçu em diferentes fases de colonização: segredo e Foz do Areia. In: Agostinho AA, Gomes LC. Reservatório de segredo: bases ecológicas para o manejo. Maringá: EDUEM; 1997. p. 275-292.

34. Cordeiro AAM, Corrêa MFM. Dinâmica das populações ictiícas naturais em lagos originados da mineração do xisto em São Mateus do Sul - PR. Curitiba: Instituto de Saneamento Ambiental da PUCPR; 1997. Relatório técnico.
35. Abilhoa V, Fontino VC, Azevedo Filho DP, Sá PZ, Acioli P, Bastos LP. Composição e estrutura da ictiofauna do reservatório do Iraí, Região Metropolitana de Curitiba, Paraná, Brasil. In: $4^{\circ}$ Seminário do Projeto Interdisciplinar de Pesquisa em Eutrofização de Águas de Abastecimento Público. 2003; Curitiba. Curitiba: Sanepar; 2003. p. 70-74.

Recebido: 31/10/2006

Received: 10/31/2006

Aceito: 04/12/2006 Accepted: 12/04/2006 